\title{
Model Building and Anisotropy of PrFeB Permanent Magnetic Materials
}

\author{
Min Liu, Xuehui Cai, Weiping Gong, Yajie Li, Lixia Cheng* \\ Guangdong Provincial Key Laboratory of Electronic Functional Materials and Devices, Huizhou College, Huizhou, China \\ Email: *lixia02741@163.com
}

How to cite this paper: Liu, M., Cai, X.H., Gong, W.P., Li, Y.J. and Cheng, L.X. (2020) Model Building and Anisotropy of PrFeB Permanent Magnetic Materials. Materials Sciences and Applications, 11, 757-766. https://doi.org/10.4236/msa.2020.1111051

Received: June 7, 2020

Accepted: November 20, 2020

Published: November 23, 2020

Copyright (c) 2020 by author(s) and Scientific Research Publishing Inc. This work is licensed under the Creative Commons Attribution International License (CC BY 4.0).

http://creativecommons.org/licenses/by/4.0/

(c) (i) Open Access

\begin{abstract}
This paper considers that the crystal grains of HDDR $\operatorname{Pr}_{2} \mathrm{Fe}_{14} \mathrm{~B}$ permanent magnetic material are cubic, the size is $0.3 \mu \mathrm{m}$, and the crystal grains are in simple cubic accumulation. It is considered that there are boundary phases between grains. It is assumed that the boundary phases are non-magnetic phases with the thickness of $d$, and evenly distributed between grains. The anisotropy expression of single grain boundary is given considering structure defect and intergranular exchange coupling interaction. Based on micro-magnetic simulation calculation, the variation of the average anisotropy of a single grain with the structural defects and boundary phases was calculated. The results show that when the thickness of structural defects is constant, the average anisotropy of a single grain decreases with increasing of grain boundary phase thickness, and while the thickness of grain boundary phase is constant, it also decreases with increasing of structural defect thickness.
\end{abstract}

\section{Keywords}

Structural Defects, Boundary Phases, Exchange Coupling Interactions, Anisotropy

\section{Introduction}

HDDR (Hydrogenation, Disproportionation, Desorption, Recombination) process is now well established as an effective process for preparing anisotropic $\mathrm{NdFeB}$ magnetic powders [1] [2] [3]. Theoretically, the structure, lattice constant, magnetocrystalline anisotropy constant, exchange integral constant and saturation magnetization of $\mathrm{Pr}_{2} \mathrm{Fe}_{14} \mathrm{~B}$ and $\mathrm{Nd}_{2} \mathrm{Fe}_{14} \mathrm{~B}$ are very close [4]. Since the intrinsic magnetic properties of $\mathrm{Pr}_{2} \mathrm{Fe}_{14} \mathrm{~B}$-type alloy are comparable to those of $\mathrm{Nd}_{2} \mathrm{Fe}_{14} \mathrm{~B}$-type alloy, recently researchers had attempted the HDDR process to 
prepare $\operatorname{Pr}_{2} \mathrm{Fe}_{14} \mathrm{~B}$-type magnetic powders with additives such as $\mathrm{Co}, \mathrm{Zr}, \mathrm{Ga}$ and $\mathrm{Nb}$ [5] [6] [7]. Han's [8] [9] investigation shows that as long as disproportionation time is reasonably controlled, the disproportionation products display a rod-like microstructure with self-organized hexagonal $\mathrm{PrH}_{2}$ nanorods embedded in Fe matrix, and the highly ordered rod-like structure is responsible for the high degree of texture orientation of HDDR $\operatorname{Pr}_{13} \mathrm{Fe}_{79.4} \mathrm{~B}_{7} \mathrm{Nb}_{0.3} \mathrm{Ga}_{0.3}$ magnetic powders. Zhong [10] adopted the modified HDDR process to prepare pure ternary anisotropy $\mathrm{Pr}_{2} \mathrm{Fe}_{14} \mathrm{~B}$-type magnetic powders. At present, there is much experimental research work on PrFeB permanent magnetic materials, and no theoretical research work has been seen. This paper attempted to establish the anisotropy theoretical model of PrFeB permanent magnet material, and further investigated the anisotropy variation of magnetic powders with structural defects and grain boundary phase. It hopes that these results of this paper can provide theoretical guidance for the experimental preparation of highly anisotropic magnetic powders.

\section{Theory Model of $\operatorname{Pr}_{2} \mathrm{Fe}_{14} \mathrm{~B}$-Type Magnetic Powders}

Assumed that the HDDR $\operatorname{Pr}_{2} \mathrm{Fe}_{14} \mathrm{~B}$ grain is a cube with size of $0.3 \mu \mathrm{m}$, and these grains are stacked in simple cubic form, as shown in Figure 1. represents a single grain, _ represents the boundary phase. The grain is affected by the exchange coupling interaction between adjacent grains. For a single crystal grain, due to its face center, the rib and the apex angle own to different contact conditions with adjacent grain, therefore, the anisotropy of three regions is also different. The plane-centered region of grains is only affected by the exchange coupling of adjacent single grains, denoted as $N=1$. The edge regions are affected by adjacent three grains, denoted by $N=3$. The top Angle regions are affected by adjacent seven grains, denoted by $N=7$.

Arcas [11] considered that a single crystal of a nano-magnet is directly connected to the surrounding $\mathrm{N}$-grain, and used this expression $K_{1}(r)=K_{1} / N^{t / 2}$ to describe anisotropy variation of grain surface. Based on the special microstructure of HDDR $\mathrm{Nd}_{2} \mathrm{Fe}_{14} \mathrm{~B}$ grains, the grain surface is affected by both exchange coupling interaction and structural defects. When the grain surface structure defect thickness $r_{0}$ is less than the exchange coupling interaction length lex/2, Liu

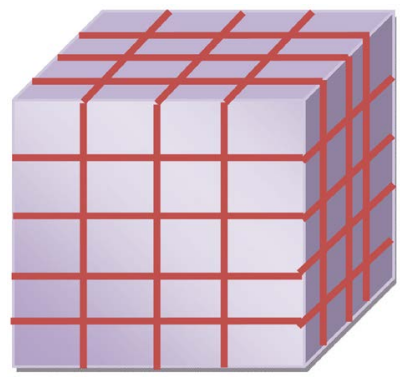

Figure 1. Grains stacking schematic diagram of $\operatorname{Pr}_{2} \mathrm{Fe}_{14} \mathrm{~B}$-type magnetic powders, represents a single grain, represents the boundary phase. 
[12] used this expression $K_{1}(r)=\left\{\begin{array}{ll}K_{1}\left\{1-\exp \left[-\left(\frac{2 r^{2}}{r_{0} l e x}\right)^{2}\right]\right\}, & 0 \leq r \leq r_{0} \\ K_{1}\left\{1-\exp \left[-\left(\frac{2 r}{l e x}\right)^{2}\right]\right\}, & r_{0} \leq r \leq \frac{l e x}{2}\end{array}\right.$ to indicate the surface anisotropy change of $\mathrm{Nd}_{2} \mathrm{Fe}_{14} \mathrm{~B}$ grain. When the surface structure defect thickness $r_{0}$ of the grain is larger than the exchange coupling interaction length lex/2, Liu [12] used this expression

$$
K_{1}(r)=\left\{\begin{array}{ll}
K_{1}\left\{1-\exp \left[-\left(\frac{2 r^{2}}{r_{0} l e x}\right)^{2}\right]\right\}, & 0 \leq r \leq \frac{l e x}{2} \\
K_{1}\left\{1-\exp \left[-\left(\frac{r}{r_{0}}\right)^{2}\right]\right\}, & \frac{l e x}{2} \leq r \leq r_{0}
\end{array}\right. \text { to represent the surface ani- }
$$

sotropy change of $\mathrm{Nd}_{2} \mathrm{Fe}_{14} \mathrm{~B}$ grain.

Not only the intrinsic magnetic properties of $\operatorname{Pr}_{2} \mathrm{Fe}_{14} \mathrm{~B}$-type alloy are comparable to those of $\mathrm{Nd}_{2} \mathrm{Fe}_{14} \mathrm{~B}$-type alloy, but also the microstructure of the $\operatorname{Pr}_{2} \mathrm{Fe}_{14} \mathrm{~B}$ magnetic powder grains is similar to that of the $\mathrm{Nd}_{2} \mathrm{Fe}_{14} \mathrm{~B}$ magnetic powder grains [9], thus, this paper considered that the change of surface anisotropy of $\mathrm{Pr}_{2} \mathrm{Fe}_{14} \mathrm{~B}$ grains is similar to that of surface anisotropy of $\mathrm{Nd}_{2} \mathrm{Fe}_{14} \mathrm{~B}$ grains. Since the grains of the $\operatorname{Pr}_{2} \mathrm{Fe}_{14} \mathrm{~B}$ magnetic powder are stacked in a simple cubic structure, The face of a single grain directly contacts with one grain (record as $N=1$ ), the ridge of a single grain directly contacts with three grains (record as $N=3$ ), and corner regions of a single grain directly contact with seven grains (record as $N=7$ ), the anisotropy change of the three regions is related to $N$, thus, the surface anisotropy $K(r)$ of $\operatorname{Pr}_{2} \mathrm{Fe}_{14} \mathrm{~B}$ grain can be rewritten as:

When the structure defect thickness $r_{0}$ of grain surface is smaller than the exchange coupling interaction length lex/2

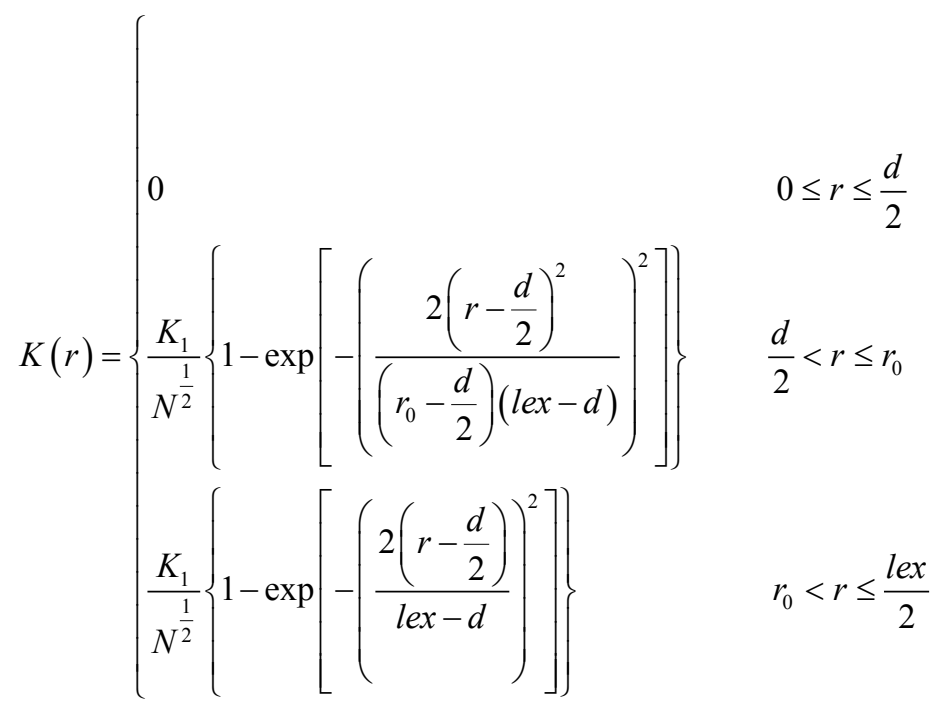

When the structure defect thickness $r_{0}$ of grain surface is larger than the exchange coupling interaction length lex/2 


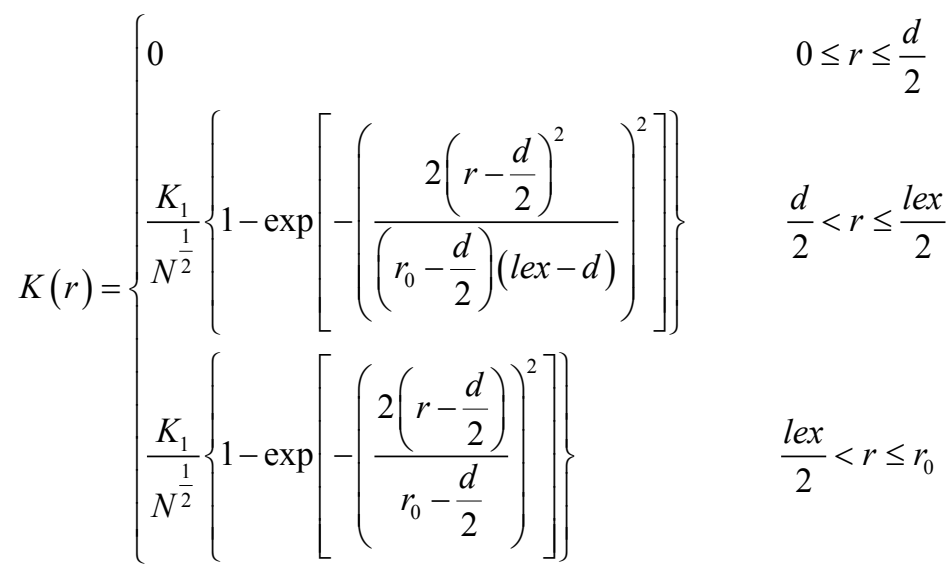

where $K_{1}$ is the normal magnetocrystalline anisotropy constant, $r_{0}$ is the structure defect thickness of grain surface, $r$ is the distance to the grain intergranular center, lex is the exchange coupling length between grains, $d$ is the boundary phase thickness.

\section{Anisotropy of $\operatorname{Pr}_{2} \mathrm{Fe}_{14} \mathrm{~B}$ Grain}

When $r_{0} \leq \frac{l e x}{2}$, the average anisotropy $\left\langle K_{i n}\right\rangle,\left\langle K_{p 1}\right\rangle,\left\langle K_{p 2}\right\rangle,\left\langle K_{p 3}\right\rangle$ of the interior, face center, ridge and corner region of a single grain can be respectively represented as:

$$
\begin{aligned}
& \left\langle K_{\text {in }}\right\rangle=\frac{2}{l e x}\left(\int_{d / 2}^{r_{0}} \frac{K_{1}}{N^{1 / 2}}\left\{1-\exp \left[-\left(\frac{2 r^{2}}{r_{0} l e x}\right)^{2}\right]\right\} \mathrm{d} r\right. \\
& \left.+\int_{r_{0}}^{l e x / 2} \frac{K_{1}}{N^{1 / 2}}\left\{1-\exp \left[1-\left(\frac{2 r}{l e x}\right)^{2}\right]\right\} \mathrm{d} r\right) \\
& \left\langle K_{p 1}\right\rangle=\frac{2}{l e x}\left(\int_{d / 2}^{r_{0}}\left\{1-\exp \left[-\left(\frac{2 r^{2}}{r_{0} l e x}\right)^{2}\right]\right\} \mathrm{d} r\right. \\
& \left.+\int_{r_{0}}^{l e x / 2} \frac{K_{1}}{N^{1 / 2}}\left\{1-\exp \left[1-\left(\frac{2 r}{l e x}\right)^{2}\right]\right\} \mathrm{d} r\right) \\
& \left\langle K_{p 2}\right\rangle=\frac{2}{l e x}\left(\int_{d / 2}^{r_{0}} \frac{K_{1}}{3^{1 / 2}}\left\{1-\exp \left[-\left(\frac{2 r^{2}}{r_{0} l e x}\right)^{2}\right]\right\} \mathrm{d} r\right. \\
& \left.+\int_{r_{0}}^{l e x / 2} \frac{K_{1}}{N^{1 / 2}}\left\{1-\exp \left[1-\left(\frac{2 r}{\text { lex }}\right)^{2}\right]\right\} \mathrm{d} r\right) \\
& \left\langle K_{p 3}\right\rangle=\frac{2}{l e x}\left(\int_{d / 2}^{r_{0}} \frac{K_{1}}{7^{1 / 2}}\left\{1-\exp \left[-\left(\frac{2 r^{2}}{r_{0} l e x}\right)^{2}\right]\right\} \mathrm{d} r\right. \\
& \left.+\int_{r_{0}}^{\operatorname{lex} / 2} \frac{K_{1}}{N^{1 / 2}}\left\{1-\exp \left[1-\left(\frac{2 r}{l e x}\right)^{2}\right]\right\} \mathrm{d} r\right)
\end{aligned}
$$


When $r_{0}>\frac{l e x}{2}$, the average anisotropy $\left\langle K_{i n}\right\rangle,\left\langle K_{p 1}\right\rangle,\left\langle K_{p 2}\right\rangle,\left\langle K_{p 3}\right\rangle$ of the interior, face center, ridge and corner region of a single grain can be respectively represented as:

$$
\begin{aligned}
&\left\langle K_{\text {in }}\right\rangle= \frac{1}{r_{0}}\left(\int_{d / 2}^{l e x / 2} \frac{K_{1}}{N^{1 / 2}}\left\{1-\exp \left[-\left(\frac{2 r^{2}}{r_{0} l e x}\right)^{2}\right]\right\} \mathrm{d} r\right. \\
&\left.+\int_{l e x / 2}^{r_{0}} \frac{K_{1}}{N^{1 / 2}}\left\{1-\exp \left[1-\left(\frac{2 r}{l e x}\right)^{2}\right]\right\} \mathrm{d} r\right) \\
&\left\langle K_{p 1}\right\rangle= \frac{1}{r_{0}}\left(\int_{d / 2}^{l e x / 2} \frac{K_{1}}{3^{1 / 2}}\left\{1-\exp \left[-\left(\frac{2 r^{2}}{r_{0} l e x}\right)^{2}\right]\right\} \mathrm{d} r\right. \\
&\left.+\int_{l e x / 2}^{r_{0}} \frac{K_{1}}{N^{1 / 2}}\left\{1-\exp \left[1-\left(\frac{2 r}{l e x}\right)^{2}\right]\right\} \mathrm{d} r\right) \\
&\left\langle K_{p 2}\right\rangle= \frac{1}{r_{0}}\left(\int_{d / 2}^{l e x / 2} \frac{K_{1}}{7^{1 / 2}}\left\{1-\exp \left[-\left(\frac{2 r^{2}}{r_{0} l e x}\right)^{2}\right]\right\} \mathrm{d} r\right. \\
&\left.\left.\left.+\int_{l e x / 2}^{r_{0}} \frac{K_{1}}{N^{1 / 2}}\left\{1-\exp \left[1-\left(\frac{2 r}{l e x}\right)^{2}\right]\right\} \mathrm{d} r\right)^{1 / 2}\left[1-\left(\frac{2 r}{l e x}\right)^{2}\right]\right\} \mathrm{d} r\right) \\
&\left\langle K_{p 3}\right\rangle= \frac{1}{r_{0}}\left(\int_{d / 2}^{l e x / 2} K_{1}\left\{1-\exp \left[-\left(\frac{2 r^{2}}{r_{0} l e x}\right)^{2}\right]\right\} \mathrm{d} r\right. \\
&+\int_{l e x / 2}^{r_{0}} \frac{K_{1}}{N^{1 / 2}}\{1-\exp [1-(1)
\end{aligned}
$$

Boundary defect zone anisotropy of $\operatorname{Pr}_{2} \mathrm{Fe}_{14} \mathrm{~B}$ grain $K_{1}^{\prime}$ can be expressed as:

$$
K_{1}^{\prime}=\frac{\left\langle K_{p 1}\right\rangle V_{1}+\left\langle K_{p 2}\right\rangle V_{2}+\left\langle K_{p 3}\right\rangle V_{3}}{V_{\text {tot }}-V_{\text {in }}}
$$

The average anisotropy $\langle K\rangle$ of a single grain can be expressed as:

$$
\langle K\rangle=\frac{6^{*}\left(\left\langle K_{p 1}\right\rangle V_{1}+\left\langle K_{p 2}\right\rangle V_{2}+\left\langle K_{p 3}\right\rangle V_{3}\right)+K_{1} V_{i n}}{V_{t o t}}
$$

where, $V_{\text {tot }}=(D+d)^{2}$.

$$
\begin{aligned}
& \text { If } r_{0} \leq \frac{l e x}{2}, V_{1}=(D+d-l e x)^{2} * \frac{l e x}{2}, V_{2}=(D+d-l e x) * l e x^{2}, V_{3}=\frac{4}{3}\left(\frac{l e x}{2}\right)^{2}, \\
& V_{\text {in }}=(D+d-l e x)^{3} . \\
& \text { If } r_{0}>\frac{l e x}{2}, V_{1}=\left(D+d-2 r_{0}\right)^{2} * r_{0}, V_{2}=\left(D+d-2 r_{0}\right) * r_{0}^{2}, V_{3}=\frac{4}{3} r_{0}^{3}, \\
& V_{\text {in }}=\left(D+d-2 r_{0}\right)^{3} .
\end{aligned}
$$

$V_{t o t}$ and $V_{i n}$ indicate the volume of a single grain and that of a grain not affected by structural defects and exchange coupling effect, respectively. $V_{1}, V_{2}$ 
and $V_{3}$ represents the volume of the face center, ridge and corner region of a single grain, respectively. The intrinsic magnetic parameter of $\operatorname{Pr}_{2} \mathrm{Fe}_{14} \mathrm{~B}$ is: $K_{1}=$ $5.6 \mathrm{MJ} / \mathrm{m}^{3}, A=7.7 \times 10^{-12} \mathrm{~J} / \mathrm{m}, \delta_{B}=3.7 \mathrm{~nm}$, Grain size $D=0.3 \mu \mathrm{m}, N_{\text {eff }}=0.6, J_{s}=$ $1.56 \mathrm{~T}$, lex $=3.7 \mathrm{~nm}$.

\section{Result and Discussion}

When the boundary phase thickness $d$ is $1 \mathrm{~nm}$ and the structure defect thickness $r_{0}$ takes different values, Figure 2 shows the dependence of anisotropy $K_{p 1}(r)$ of the face center region with the distance $r$ to the center of boundary phase, and Figure 3 shows that the dependence of ridge region anisotropy $K_{p 2}(r)$ on $r$, the variation of the corner region anisotropy $K_{p 3}(r)$ with $r$ is shown in Figure 4. When the structure defect thickness $r_{0}$ is constant, $K_{p 1}(r), K_{p 2}(r), K_{p 3}(r)$ all increase with increasing of $r$. This illustrates that the closer to the grain center, the bigger anisotropy of the grain face center region, the ridge region and the corner region. It also shows that with decreasing of $r_{0}$, the faster decrease rate of $K_{p 1}(r), K_{p 2}(r), K_{p 3}(r)$ with $r$, this belongs to with decrease of $r_{0}$, the change range of anisotropy from $K_{1}$ to zero is narrowing, so the variation rate of $K_{p 1}(r), K_{p 2}(r), K_{p 3}(r)$ with $r$ is faster.

When the grain structure defect thickness is $4 \mathrm{~nm}$ and the grain boundary phase thickness $d$ takes different values, Figure 5 indicates that the face center region anisotropy $K_{p 1}(r)$ varies with $r$. and Figure 6 shows that the dependence of ridge region anisotropy $K_{p 2}(r)$ on $r$, the variation of the corner region anisotropy $K_{p 3}(r)$ with $r$ is shown in Figure 7. The figures show that when $d$ takes different values, $K_{p 1}(r), K_{p 2}(r), K_{p 3}(r)$ all decrease with reducing of $r$. This indicates that the closer to the center of grain boundary phase, the smaller the anisotropy. The data in the figure further shows that the decrease rate of $K$ with $r$ in $0<r<1.85$ is greater than that of $K$ with $r$ in $1.85<r<4$, because the anisotropy is influenced by exchange-coupled affect and structure

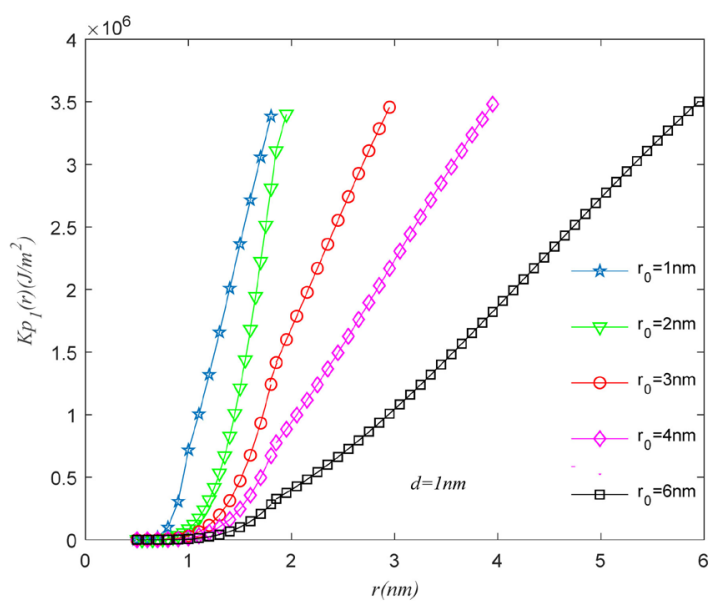

Figure 2. For the boundary phase thickness $d$ of $1 \mathrm{~nm}$ and the structure defect thickness takes different values, the dependence of anisotropy $K_{p 1}(r)$ of the face center region with the distance $r$ to the center of grain boundary phase. 


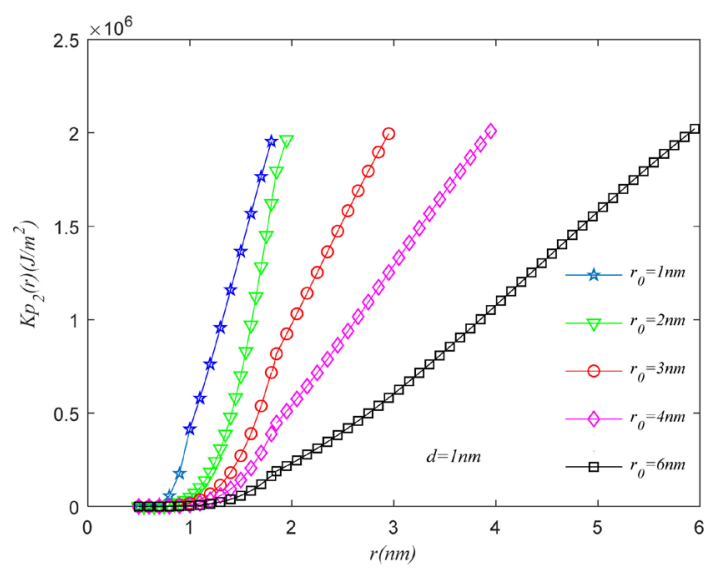

Figure 3. For the boundary phase thickness $d$ of $1 \mathrm{~nm}$ and the structure defect thickness takes different values, the dependence of ridge region anisotropy $K_{p 2}(r)$ on $r$.

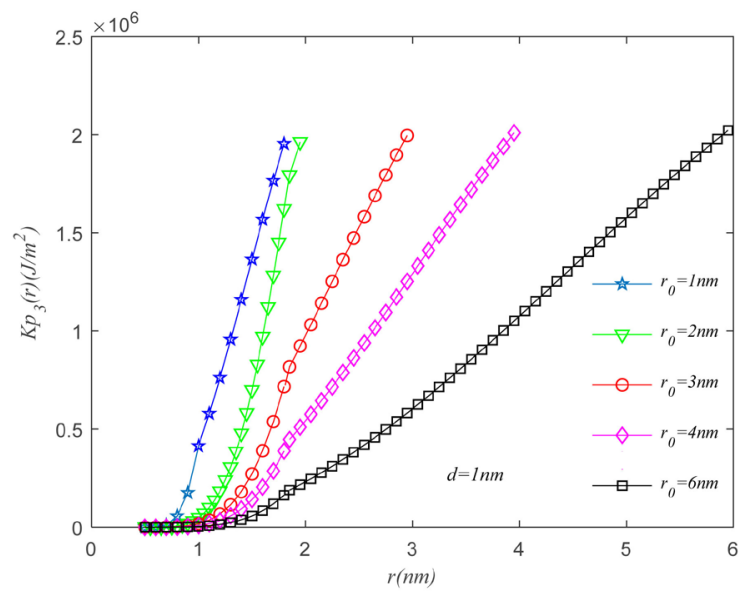

Figure 4. For the grain boundary phase thickness $d$ of $1 \mathrm{~nm}$ and the structure defect thickness takes different values, the variation of the corner region anisotropy $K_{p 3}(r)$ with $r$.

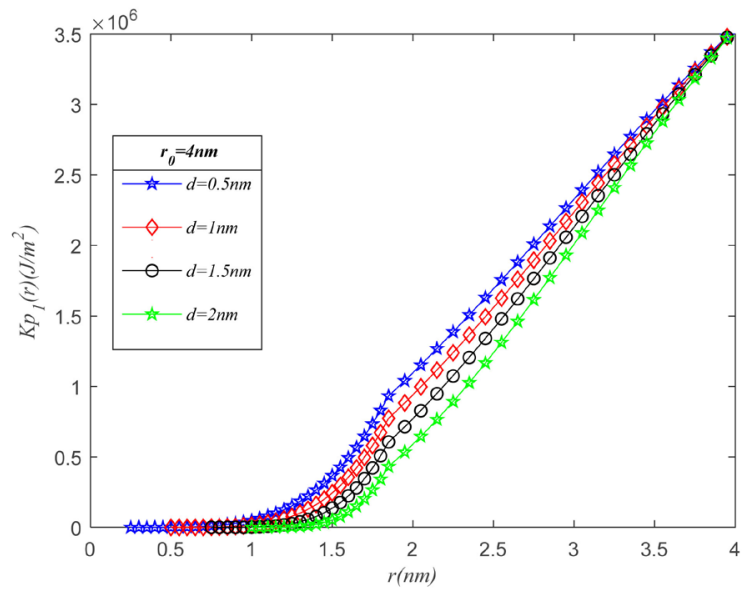

Figure 5. For the grain structure defect thickness of $4 \mathrm{~nm}$ and the grain boundary phase thickness $d$ takes different values, the change of the face center region anisotropy $K_{p 1}(r)$ with $r$. 
defects in $0<r<1.85$, But in $1.85<r<4$, the anisotropy is only affected by structural defects. Figure 8 shows the variation of material average anisotropy $\langle K\rangle$ with structure defect thickness $d$. This figure indicates that for $r_{0}$ taking different values, $\langle K\rangle$ decrease with increasing of $d$.

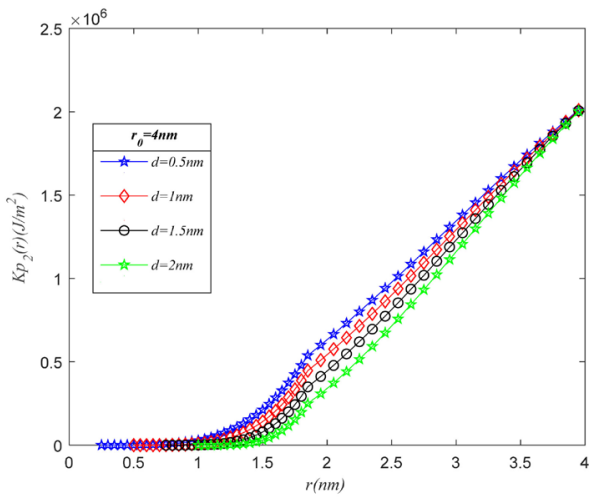

Figure 6. For the grain structure defect thickness of $4 \mathrm{~nm}$ and the grain boundary phase thickness $d$ takes different values, the dependence of ridge region anisotropy $K_{p 2}(r)$ on $r$.

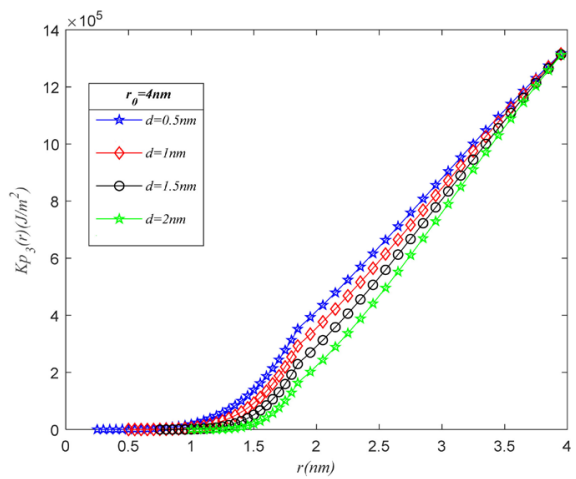

Figure 7. For the grain structure defect thickness of $4 \mathrm{~nm}$ and the grain boundary phase thickness $d$ takes different values, the variation of the corner region anisotropy $K_{p 3}(r)$ with $r$.

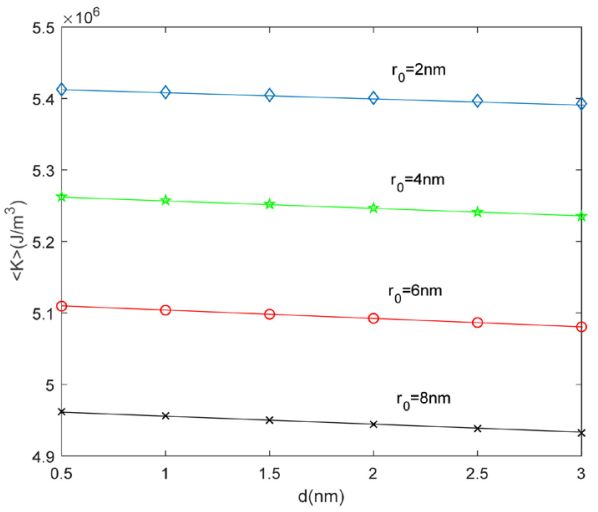

Figure 8. For different values of $r_{0}$, the variation of average anisotropy $\langle K\rangle$ of a single grain with structure defect thickness $d$. 


\section{Conclusion}

This paper investigates the effects of exchange coupling interactions and structural defects on the anisotropy of a single grain. The results show that both structure defects and exchange coupling interactions affect the anisotropy of single grains. When the thickness of structural defects is constant, the average anisotropy of a single grain decreases with increasing of grain boundary phase thickness, and while the thickness of grain boundary phase is constant, it also decreases with increasing of structure defect thickness.

\section{Acknowledgements}

The work is supported by the National Natural Science Foundation of China (Grant No. 51602376, 51602121), Guangdong Nature Science Foundation (Grant No. 2017A030310665), Natural Science Foundation of Huizhou College (Grant No. 2015167, hzuxl201626).

\section{Conflicts of Interest}

The authors declare no conflicts of interest regarding the publication of this paper.

\section{References}

[1] Gutfleischdag, O., Kirchner, A., Grünberger, W., Hinz, D., Nagel, H., Thompson, P., Chapman, J.N., Müller, K.H., Schultz, L. and Harrisdag, I.R. (1998) Textured NdFeB HDDR Magnets Produced by Die-Upsetting and Backward Extrusion. Journal of Physics D: Applied Physics, 5, 201-204.

[2] Liu, M. (2014) Anisotropy of Ternary and (Zr, Ga) Addition Magnetic Powders. Journal of Atomic and Molecular Sciences, 5, 338. https://doi.org/10.4208/jams.062914.081914a

[3] Liu, M., Gong, W.P., Li, Y.J., et al. (2018) Effect of SC Process on Microstructure of $\mathrm{Nd}_{9.8} \mathrm{Dy}_{2.4} \mathrm{Fe}_{71.8} \mathrm{Co}_{10} \mathrm{~B}_{6}$ Magnetic Powders. Modern Physics, 8, 1-4. https://doi.org/10.12677/MP.2018.84018

[4] Herbst, J.F. (1991) $\mathrm{R}_{2} \mathrm{Fe}_{14} \mathrm{~B}$ Materials: Intrinsic Properties and Technological Aspects. Reviews of Modern Physics, 63, 819-821. ttps://doi.org/10.1103/RevModPhys.63.819

[5] Faria, R.N., Brown, D.N. and Harris, I.R. (2000) The Influence of Alloying Additions and Process Parameters on the Magnetic Properties of PrFeB-Based Bonded Magnets. Journal of Alloys and Compounds, 296, 219-222.

https://doi.org/10.1016/S0925-8388(99)00535-6

[6] Saccone, F.D., Rodriguez, C.E., Sanchez, F.H. and Gutfleisch, O. (2002) Observation of Hydrogen Induced Intermediate Borides in PrFeB Based Alloys by Mössbauer Effect spectroscopy. Physica B, 320, 312-314. https://doi.org/10.1016/S0921-4526(02)00724-X

[7] Cannesan, N., Brown, D.N., Williams, A.J. and Harris, I.R. (2001) The Production and Characterisation of Highly Anisotropic PrFeCoB-Type HDDR Powders. Journal of Magnetism and Magnetic Materials, 233, 209-211. https://doi.org/10.1016/S0304-8853(01)00212-8

[8] Han, J.Z., Liu, S.Q., Du, H.L., Yang, J.B. and Yang, Y.C. (2005) Anisotropic Ternary 
$\operatorname{Pr}_{13} \mathrm{Fe}_{80} \mathrm{~B}_{7}$ Powders Prepared by Hydrogenation Disproportionation Desorption Recombination Process. Journal of Applied Physics, 97, 10F305-07. https://doi.org/10.1063/1.1852334

[9] Han, J.Z., Liu, S.Q., Wang, C.S., Chen, H., Du, H.L. and Yang, Y.C. (2009) Effects of the Conventional HDDR Process and the Additions of Co and $\mathrm{Zr}$ on Anisotropy of HDDR Pr-Fe-B-Type Magnetic Materials. Journal of Magnetism and Magnetic Materials, 321, 1331-1333. https://doi.org/10.1016/j.jmmm.2008.10.048

[10] Lin, Z., Han, J.Z. and Liu, S.Q, (2014) Coercivity Enhancement in $\operatorname{Pr}_{9.5} \mathrm{Fe}_{83} \mathrm{Zr}_{2} \mathrm{~B}_{5.5}$ Magnetic Nanomaterials. Journal of Applied Physics, 111, 07A722-24.

[11] Arcas, J., Hernando, A., Barandiara, J.M., et al. (1998) Soft to Hard Magnetic Anisotropy in Nanostructured Magnets. Physical Review B, 58, 5193-5196.

https://doi.org/10.1103/PhysRevB.58.5193

[12] Liu, M., Sun, Y., Han, G.B., Wu, Y. and Gao, R.W. (2008) Effect of Microstructure on the Coercivity of HDDR Nd-Fe-B Permanent Magnetic Alloy. Science China Physics, Mechanics \& Astronomy, 51, 1557-1559.

https://doi.org/10.1007/s11433-008-0159-6 